\title{
Wireless Energy Transfer by Means of Inductive Coupling for Dairy Cow Health Monitoring
}

\author{
Ben Minnaert ${ }^{\mathrm{a}, *}$, Bart Thoen ${ }^{\mathrm{a}}$, David Plets ${ }^{\mathrm{b}}$, Wout Joseph ${ }^{\mathrm{b}}$, Nobby Stevens ${ }^{\mathrm{a}}$ \\ ${ }^{a}$ Research Group DraMCo, ESAT, Technology Campus Ghent, KU Leuven, Belgium \\ ${ }^{b}$ Department of Information Technology, IMEC-Ghent University, Ghent, Belgium
}

\begin{abstract}
The increase of herd sizes hinders the capability of the dairy farmer to timely detect illnesses. Therefore, automatic health monitoring systems are deployed, but due to their high energy consumption, the application possibilities remain limited. In this work, a wireless, inductive charging solution for dairy cow monitoring is designed. This system is mounted at the eating trough, and the amount of energy transferred each eating turn is determined experimentally. For the first time, inductive wireless power transfer is used to charge on-body sensor networks for cattle. Measurements at a research farm on 40 dairy cows show an average energy transfer of $96 \mathrm{~J}$ per meal, for an average eating time of $160 \mathrm{~s}$. It is demonstrated that inductive power transfer is a viable technology to resolve the energy provision challenge for the automatic and real-time health monitoring of dairy cows.

Keywords: automation, dairy cows, energy harvesting, health monitoring systems, inductive charging, on-body sensors, wireless power transfer, wireless sensor networks.
\end{abstract}

\section{Introduction}

Dairy farmers aim at increasing their herd size, either out of necessity to survive in a cost competitive market or to generate more profits [1]. The more dairy cows on the farm, the more milk can be produced per euro of investment,

${ }^{*}$ Corresponding author. Email address: ben.minnaert@kuleuven.be 
5 leading to a lower relative cost [2]. This desire to increase the herd size on a farm can be seen in the numbers: in the United States, the average dairy cow herd size increased by $325 \%$ between 1980 and 2004 [3]. Also in the European Union, the number of cows per farm is increasing with a growth of $30 \%$ between 2007 and 2010 [4].

The total cost of milk production consists of many different components, e.g., machinery, land costs, veterinary costs, buildings, animal purchases,... By far, the two most expensive components are feed and labor costs [5]. An increasing farm size does not necessarily guarantee a lower cost per unit of produced milk since the associated labor cost can cancel out the added cost reduction. Indeed, if the herd size is limited, the farmer has the ability to individually follow up all the cows frequently. However, the larger the herd, the more labor intensive and less practical it becomes for the farmer to monitor all the dairy cows. Nevertheless, a strict monitoring of all cows remains necessary to timely detect anomalies in the health of the farm animals as a late detection may lead to significant costs. For example, a cost of at least 150 euro is associated with a missed case of mastitis or per missed calving and 250 euro or more per missed heat or per late detection of lameness [6, 7].

To manage the increasing herd size in an economically efficient way, the farmer can rely on automatic health monitoring systems for the collection and 25 interpretation of animal data. Even for farms with less than a hundred dairy cows, automatic animal monitoring can be economically beneficial since it reduces the associated labor [2].

Automatic monitoring systems can be implemented for the detection of illnesses, predicting the calving moment, and tracking the movement and location of the animal [8, 9, 10, 11, 12]. On-body sensors allow measuring different parameters of the animal, which can be wirelessly transferred to a back-end server for data interpretation [13, 14]. The back-end system can, when a possible anomaly is detected, alert the farmer through portable electronics, e.g., the farmer's smartphone (Fig. 1). A timely detection and reliable interpretation of the data requires a near-real-time collection and processing of the measurements. 


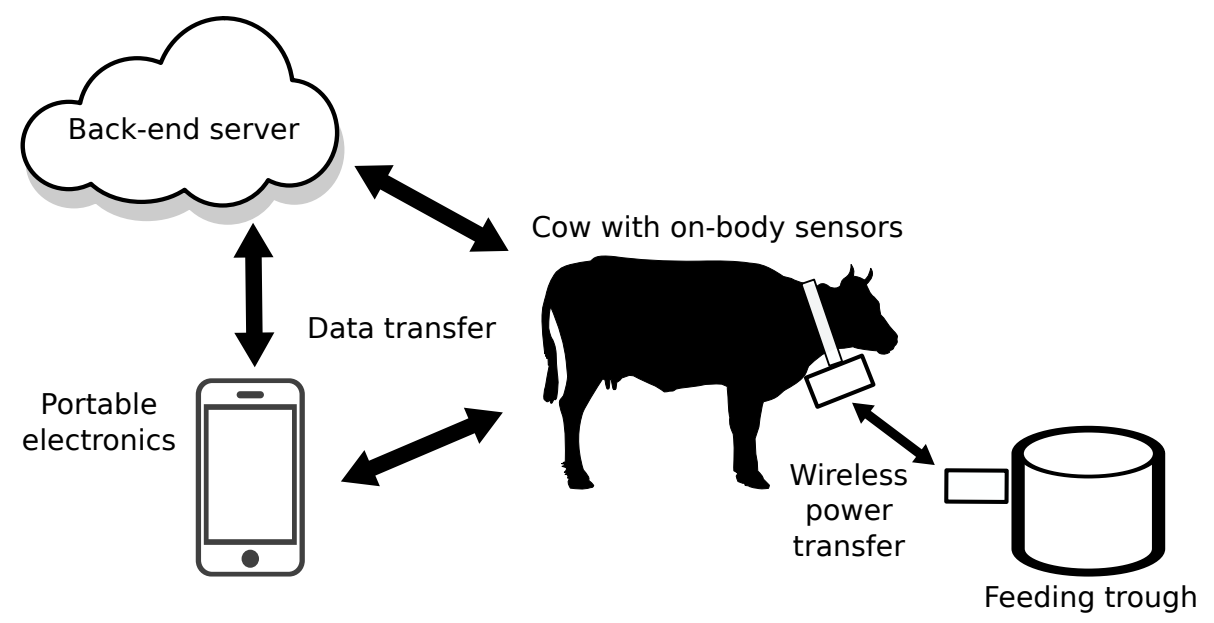

Figure 1: On-body sensors measure different parameters of the animal, which are wirelessly transferred to a back-end server for data interpretation. The back-end system and the onbody system can share its information with the farmer's portable electronics and e.g., alert the farmer when a possible anomaly is detected. The on-body health system is wirelessly charged at a feeding trough. 


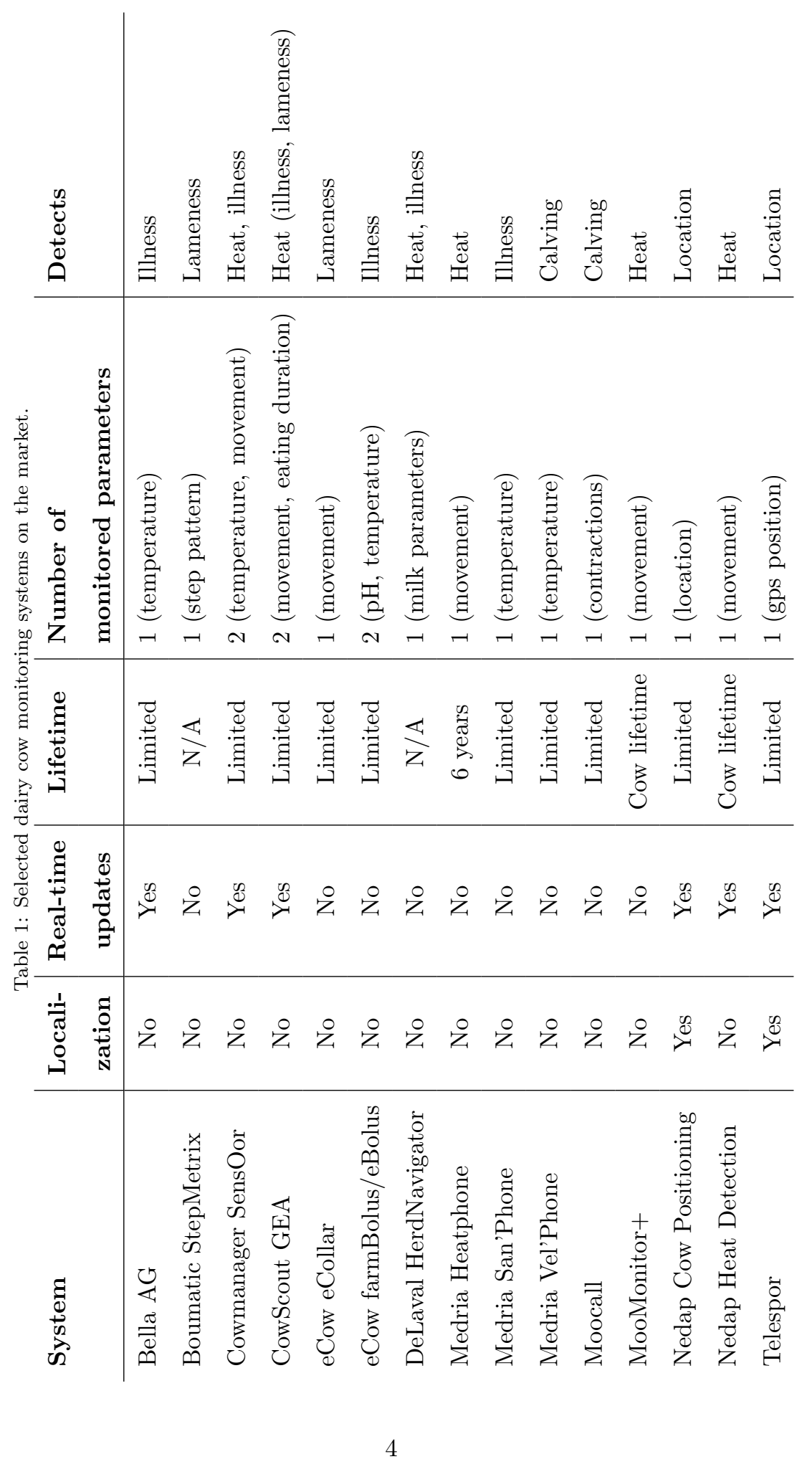


Table 1 lists several important animal monitoring systems available on the market, the parameters they monitor and which anomalies they detect. The systems listed in the table all monitor only one or two parameters at once, often not in real-time. None of them combine multi-parameter information. 40 Medria, eCow and Nedap have the expertise to monitor multiple parameters (e.g., movement, temperature, or location), but these features require separate systems (e.g., Heatphone, San'Phone and Vel'Phone). An integrated animal monitoring system which is able to detect several different parameters as illness, calving, movement and location at once currently not exists. This requires the farmer to buy and integrate different measurement solutions.

An important barrier for an integrated system is the high energy consumption. Indeed, powering different accurate sensors and wirelessly transferring the data in real-time to a back-end server requires a significant amount of energy. Even when only one or two parameters are measured, the lifetime of current devices are often limited. Solutions that claim a lifetime equal to the cow's lifetime have to focus on only one monitored parameter (Table 1). Therefore, in a lot of systems, the farmer has to manually replace the battery every few months or every year. This contradicts with the objective of a maintenance-free, automatic health system to reduce the labor cost.

55 A solution to the above problems is wirelessly charging the monitoring system at the drinking or eating trough by inductive coupling (Fig. 1). In this way, the system can wirelessly receive enough energy every day to continue operation. As a result, not only more energy can be made available to the system, allowing the real-time measurement of multiple parameters, but the system allows for a maintenance-free solution during the entire lifetime of the cow, under the condition that the lifetime of the sensors (including their reliability and accuracy) is sufficiently large. Moreover, the wireless charging avoids the regular replacement of single-use batteries, leading to a reduced impact on the environment.

By installing a transmitter coil at an eating trough and a receiver coil in the 65 collar (which can serve as a central hub for on-body sensors), wireless power transfer can be realized during the eating time slots at a dairy farm. Measure- 
ments were performed at a dairy farm on 40 lactating cows to experimentally determine how much power transfer can be expected through inductive coupling every time the cow eats. This allowed to determine the daily energy transfer, leading to an evaluation of the feasibility of using inductive coupling as a way to wirelessly charge automatic on-body health systems for dairy cows. The main novelty of this work is that, for the first time, inductive wireless power transfer was applied to charge on-body sensor networks on cattle.

The paper is organized as follows: in Section 2, the principle and background for wireless inductive charging is described. In Section 3 , the methodology for our setup is discussed. Finally, the results of the field tests with dairy cows can be found in Section 4

\section{Inductive wireless charging}

To wirelessly charge the system, the principle of inductive coupling is applied: an alternating current through a transmitter coil generates a time-varying magnetic field (Fig. 2a). This field generates an alternating voltage in a receiver coil, thus enabling energy transfer from the transmitter to the receiver coil, located in the collar of the cow.

Inductive wireless power transfer has already entered the market, as well for low power (e.g., electronic portable devices) as higher power applications (e.g., electrical vehicles) [15, 16, 17. However, the devices on the market are all static and deterministic. This means that the position of the receiver with regard to the transmitter is defined and unaltered over the charging time, resulting in a constant inductive coupling.

Applications for non-static wireless power transfer applications, where the relative transmitter-receiver positions are highly time-variant and randomized during the charging process, have not yet penetrated the market. Research on these applications is less mature. Some examples are the charging of electrical cars while driving [18, moving vehicles (e.g., automated guided vehicles or 95 drones) [19, 20], or moving electronic portable devices [21, 22]. 


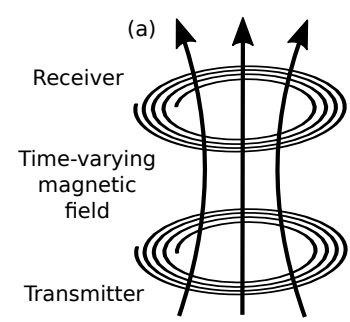

High power transfer

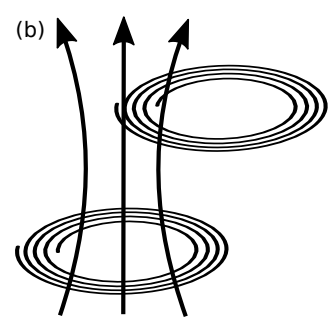

Low power transfer

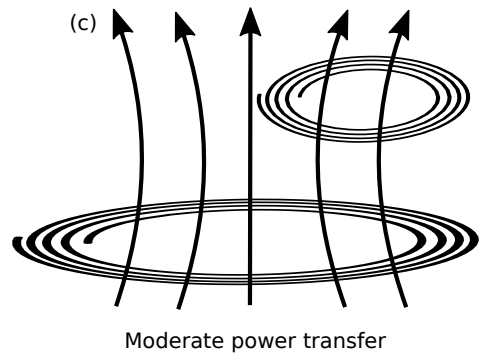

Figure 2: The principle of inductive coupling: a transmitter and receiver coil are coupled through a magnetic field. (a) If the transmitter and receiver coil are identical and perfectly aligned, a large portion of the transmitted magnetic flux can be captured by the receiver, resulting in a high power transfer. (b) If there is a significant lateral mismatch between the transmitter and receiver coil, only a small part of the magnetic flux is captured by the receiver, leading to a low power transfer (c) The same lateral displacement as in (b) leads to a higher power transfer if an elongated oval transmitter is used.

For this application, the coupling between transmitter and receiver is highly variable in time. Non-static wireless power transfer differs from a static system in three areas.

- The vertical distance between the transmitter and receiver coil is variable, and ranges from a few $\mathrm{cm}$ to tens of $\mathrm{cm}$, whereas in a static ideal system, the vertical distance is constant and small, often only 5 to $10 \mathrm{~mm}$.

- The lateral position between the transmitter and receiver is variable, resulting in an additional circumstance that contributes to a variable coupling. Sometimes, the coils are well-aligned, leading to a reasonable coupling (Fig. 2a), but often, the lateral alignment is not optimal (Fig. 2 b). Obviously, for a static ideal configuration, the transmitter and receiver coils are not only close to be perfectly aligned, but also the coupling remains constant.

- Finally, the receiver can be tilted, changing the angle between the transmitter and receiver plane.

In this study, a non-static, inductive charging system is proposed and exper- 
imentally investigated, installed on the collar of a cow. As far as we know, this is the first time inductive charging is applied for dairy cows. More specifically:

- The optimal wireless power transfer setup for this application was determined.

- The average amount of power transfer during one eating turn was measured.

- The energy transfer per day was determined by experiments with cows, allowing for an evaluation of the feasibility of wireless power transfer for on-body dairy cow sensors.

\section{Methodology}

$\mathrm{X}$

\subsection{Location}

The question arises where the on-cow system can be most easily charged. It must be a location the dairy cow visits often, preferably at least once a day. Different options are possible: the drinking trough, the forage feeding box, the concentrate feeding box or the automatic milking system. The first two options have the disadvantage that the cow often has a lot of lateral freedom to move. This complicates the charging, since multiple transmitter coils would be needed to ensure that the receiver coil of the cow is located near the magnetic field of the transmitter coil. Moreover, it would require a mechanism to detect to which transmitter coil the cow is the closest in order to activate that specific coil. Furthermore, at a farm, the dairy cows often have the choice between more than one drinking trough or forage feeding box. Therefore, the two best options are the concentrate feeding box and the automatic milking system. In both options, the cow often has limited ability to move laterally, due to the presence of a railing. Also, the number of concentrate boxes and automatic milking systems at a farm is small, requiring only a couple of transmitter systems installed per farm, reducing the system cost for the farmer. 


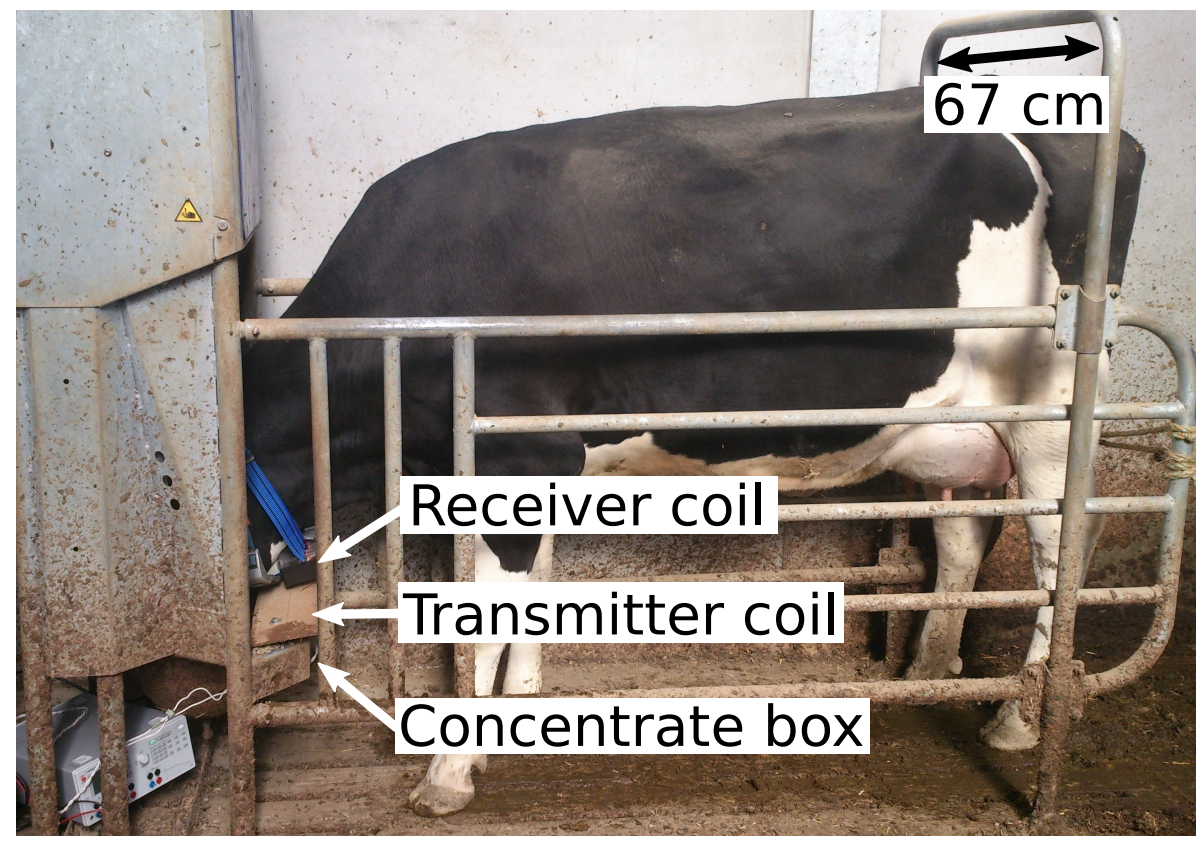

Figure 3: A cow, equipped with the wireless power transfer system with inductive coupling, is eating at the concentrate feeding box at the Research Institute for Agriculture, Fisheries and Food Research. A transmitter coil is installed at the concentrate feeding box. When the cow eats, energy is transferred wirelessly to a receiver coil, located at the collar of the dairy cow.

For this work, a common concentrate feeding box was used for the field measurements (Fig. 3), but analogous results can be achieved with the automatic milking system. The concentrate box is located in a research dairy farm at the Research Institute for Agriculture, Fisheries and Food Research in Melle (ILVO), Belgium. The railing has a width of $67 \mathrm{~cm}$ and allows for a single cow to eat. The circular feeding bowl of the box has a diameter of $40 \mathrm{~cm}$. A transmitter coil, installed at the concentrate feeding box, allows the wireless charging of the receiver in the collar of the cow when eating. A detection mechanism is required to ensure the transmitter is only transmitting high power when a cow is present.

\subsection{The transmitter and receiver circuitry}

Fig. 4 shows a schematic overview of the transmitter. A potentiometer allows tuning the frequency over a broad range. An operating frequency of 


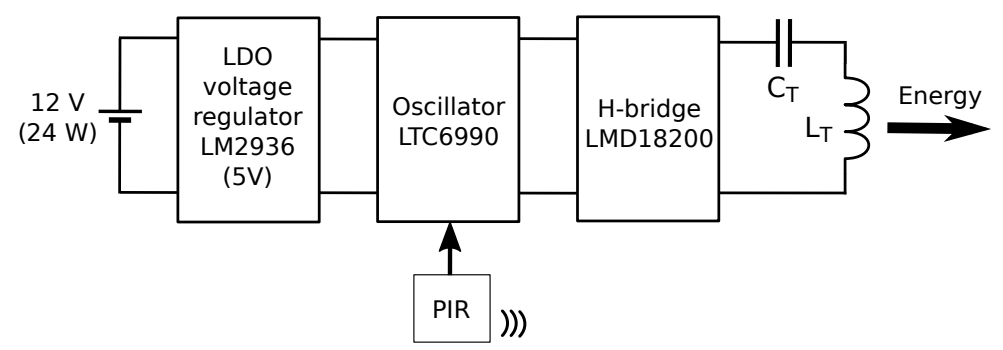

Figure 4: Schematic overview of the transmitter circuit.

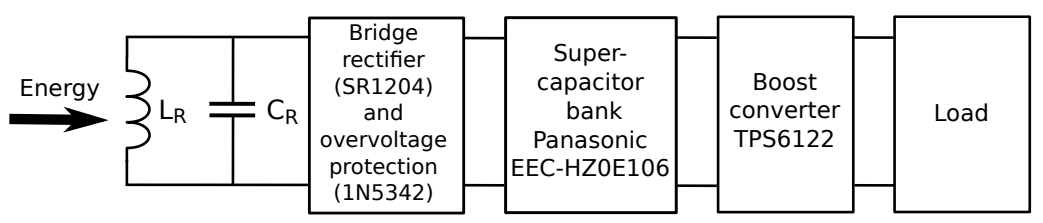

Figure 5: Schematic overview of the receiver circuit.

$90 \mathrm{kHz}$ is applied. The efficiency of power transfer is increased by adding a resonance capacitance $C_{T}$. A series resonant topology is preferable given the small AC resistance 23. The shape and dimensions of the transmitter coil $L_{T}$ are discussed in section 3.3

An off-the-shelf passive infrared (PIR) motion detector (HC-SR501) is connected to the transmitter. It was installed at the side of the concentrate box, pointing to the head of the animal, at a height of $70 \mathrm{~cm}$. The transmitter becomes active as soon as the PIR detects the presence of a cow and shuts down when during an adjustable time (for our tests set at $50 \mathrm{~s}$ ) no movement is detected.

The transmitter coil is secured onto a wooden surface and is attached, nearly horizontally, just before the circular feeding bowl, at a height of $43 \mathrm{~cm}$ from the floor. The depth of the circular feeding bowl is $25 \mathrm{~cm}$. In this way, the distance between the transmitter and receiver coil is minimized when the cow eats as is illustrated in Fig. 3

The receiver (Fig. 5) is located at the bottom of a box, attached to the collar of the cow. The efficiency is increased by adding a resonance capacitance $C_{R}$ 
in parallel, best suited for this configuration [23]. A preparatory study 24 Agilent $^{\text {TM }} 4285 \mathrm{~A} \mathrm{LCR} \mathrm{meter} \mathrm{operating} \mathrm{at} 90 \mathrm{kHz}$. A capacitance $C_{R}$ of $633 \mathrm{nF}$ in parallel with the receiver coil is applied, resulting in a resonance frequency of $90 \mathrm{kHz}$.

Not only the fencing, but the entire concentrate box is constructed out of metal (Fig. 3). This is disadvantageous for the wireless power transfer, since a changing magnetic field generates eddy currents within conductors, resulting in additional losses [25]. It is well-known that this effect can be prevented by shielding the system with a ferrite layer [25, 26. The ferrite layer will on the one hand shield the magnetic flux from the metal, and will on the other hand guide 195 the magnetic field lines in the preferable direction. A ferrite layer is applied below the transmitter coil and a ferrite core to the receiver (Fig. 6) to increase performance.

The transmitter coil is constructed from the same single isolated $\mathrm{Cu}$ wire 


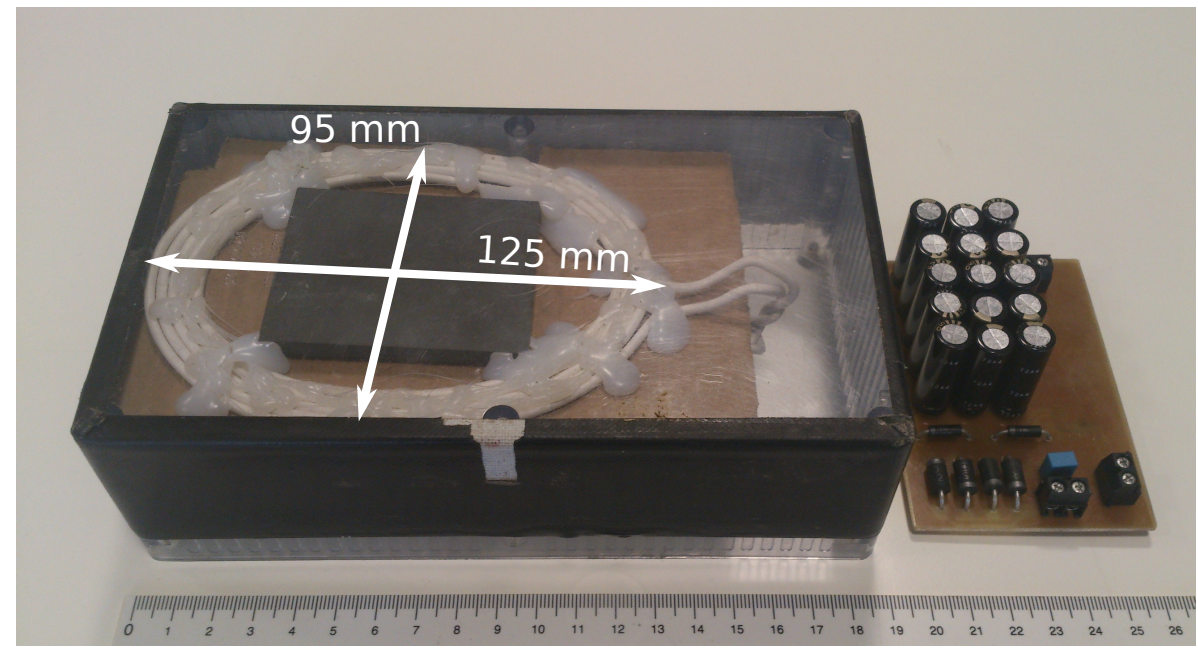

Figure 6: On the left, the bottom of the receiver box, containing the receiver coil with a ferrite core. On the right, the receiver's circuit with the supercapacitor bank.

with a cross section of $1.5 \mathrm{~mm}^{2}$. The shape of the concentrate feeding box allows in practice only for a planar coil. Two options are possible.

The first option is a nearly circular transmitter coil with the same dimensions as the receiver coil (Fig. 6). For static applications, this approach is often applied, since the more the transmitter and receiver coil match in dimensions, the higher the possible power transfer. However, for non-static applications, this approach is not necessarily optimal. The disadvantage is that, even though the power transfer is maximized with this configuration, this maximum is only achieved when the receiver coil is nicely aligned with the transmitter coil, at the center of the concentrate feeding box, as shown schematically in Fig. 22a. If there is a lateral mismatch between the transmitter and receiver coil, as shown in Fig. 2 $\mathrm{b}$, the power transfer drops significantly.

The second option is to choose for a long elongated transmitter coil, spread out over a large portion of the concentrate box. In this way, a lateral displacement of the receiver coil (as shown schematically in Fig. 25) will still allow for power transfer, although the total power transfer will be less as for optimally 215 positioned identical coils. 


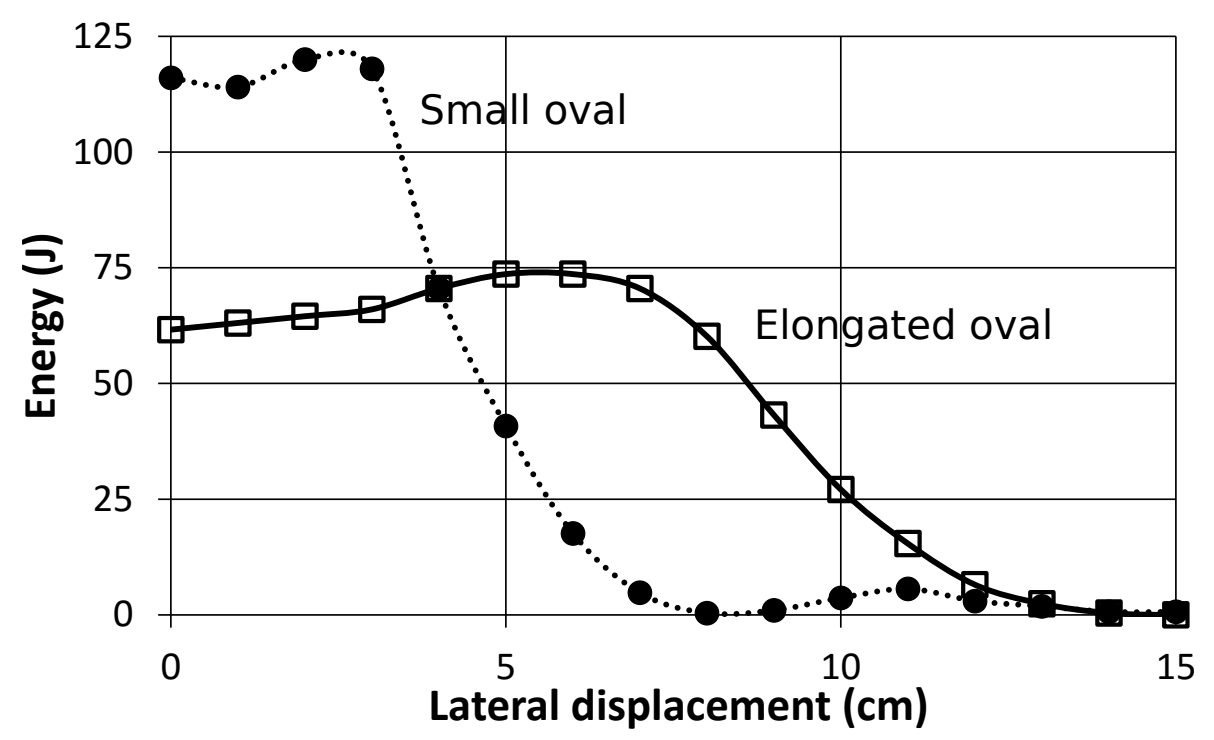

Figure 7: The amount of transferred energy in $15 \mathrm{~s}$ for a small and elongated oval transmitter, for different lateral displacements, measured from the center.

To determine the optimal choice, the two configurations were built to perform measurements. The first transmitter coil had the same dimensions as the receiver coil, i.e. a small oval planar coil with 5 exterior turns, external dimensions of $125 \mathrm{~mm} \times 95 \mathrm{~mm}$ and an inductance and quality factor of $7.7 \mu \mathrm{H}$ and 87, respectively (Fig. 2 a , b). The second configuration used an elongated 5-turn oval coil with external dimensions of $270 \mathrm{~mm}$ x $135 \mathrm{~mm}$ and an inductance and quality factor of $15 \mu \mathrm{H}$ and 170, respectively (Fig. 22). The resonance capacitors were chosen such that the resonance frequency is about $90 \mathrm{kHz}$ for both configurations. At resonance frequency, the amount of energy transferred to the supercapacitors was measured as function of time. For both configurations, the lateral position of the receiver to the transmitter was varied along the longitudinal axis of the transmitter. The vertical distance between transmitter and receiver was kept fixed at $2.0 \mathrm{~cm}$. Ferrite was present at the transmitter and receiver side. Fig. 7 shows the results, where zero lateral distance indicates that the receiver is positioned at the center of the transmitter coil.

For the first configuration (the small oval, Fig. 7), a lateral displacement 
from 0 to $3 \mathrm{~cm}$ does not significantly change the energy transfer: in about $15 \mathrm{~s}$, $120 \mathrm{~J}$ of energy is wirelessly transferred to the receiver in this setup. For larger lateral displacements, the energy transfer decreases, e.g., if the receiver is $7 \mathrm{~cm}$ shifted from the center of the transmitter, only $5 \mathrm{~J}$ is transferred in $15 \mathrm{~s}$. For the second configuration with a much more elongated oval transmitter, a lateral displacement from 0 to $8 \mathrm{~cm}$ does not considerably change the energy transfer (Fig. 7): in $15 \mathrm{~s}, 60$ to $74 \mathrm{~J}$ of energy is wirelessly transferred to the receiver. As was expected, the largest energy transfer can be achieved by using the first configuration in the condition of good alignment. These measurements indicate that if the lateral displacement remains limited to about $4 \mathrm{~cm}$, a small oval transmitter is preferable. Otherwise, an elongated oval transmitter is preferred.

\section{Field tests}

\subsection{Preparatory measurements}

245

Preparatory field measurements were performed on the dairy farm ILVO. The receiver was attached at the collar of different cows (Fig. 3). The time the cow eats at the concentrate was manually timed for 32 eating turns. An average of $142 \mathrm{~s}$ was found, with a standard deviation of $95 \mathrm{~s}$. The average energy transfer per eating turn for the different preparatory field measurements was $0.2 \mathrm{~W}$. It was visually noticed that during eating at the concentrate box, the lateral distance between the transmitter and receiver was in the majority of time more than $5 \mathrm{~cm}$. It was quite obvious from the field tests that an elongated transmitter is needed to optimize the energy transfer. The lateral position of the receiver is simply too variable to ensure an optimal power transfer with a small transmitter.

During this field test, it was also noticed that the receiver coil in the collar is mostly slightly tilted (about 10 degrees) with respect to the horizontal plane. Therefore, the transmitter coil was tilted to 10 degrees to the horizontal plane in order to achieve a better alignment with the receiver (Fig. 8). 


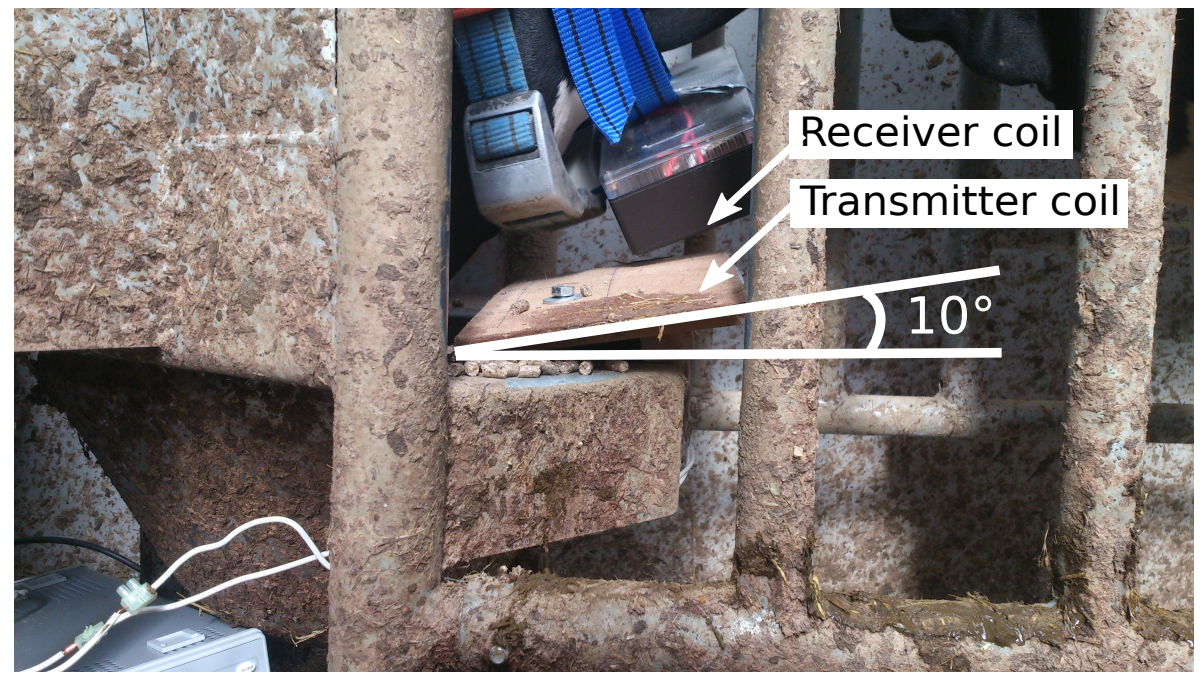

Figure 8: The transmitter coil is 10 degrees tilted with respect to the horizontal plane. This figure shows a snapshot where the receiver is not optimally oriented to the transmitter, even though the cow is eating.

\subsection{Extensive field tests}

After these initial optimizations, extensive field experiments were conducted at the farm. The transmitter was installed with the above specifications at the concentrate feeding box (Fig. 3 and 8). The receiver was attached at the collar of the cow. Each experiment consisted of one eating turn of a cow. A data logger registered every second the voltage over the supercapacitors, which is a measure for the energy captured by the system. 40 measurements with different lactating Holstein-Friesian cows were performed. This sample size enabled us to make reliable estimates for the average values of the data [27]. Each measurement started at the moment the first energy transfer occurs, and ended when the cow left the concentrate feeding box.

Fig. 9 shows one of the best measurements with respect to maximal energy transfer. The energy transferred to the supercapacitor bank is plotted as function of time. In this specific measurement, $168 \mathrm{~J}$ was transferred in $63 \mathrm{~s}$, which corresponds to an average power transfer rate of $2.67 \mathrm{~W}$. However, the wireless power transfer is not constant during time. When the cow eats, both 


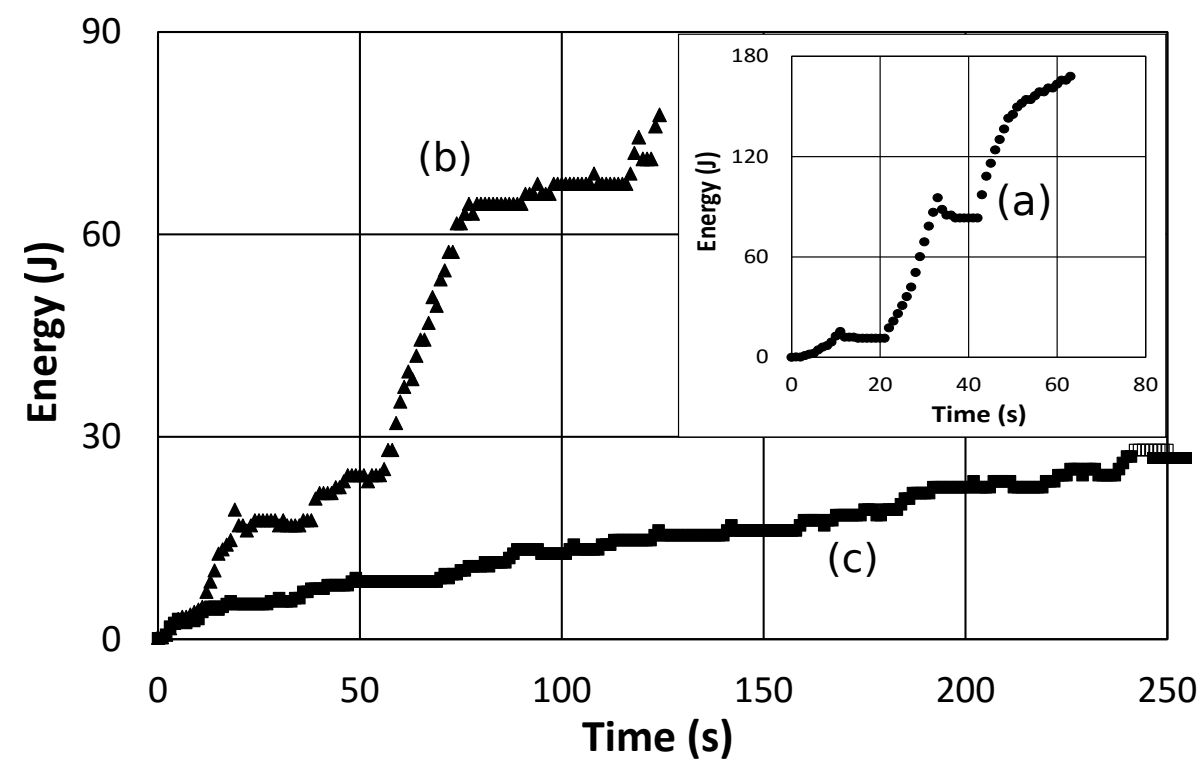

Figure 9: The amount of transferred energy as function of time: example of a measurement with (a) high, (b) moderate and (c) low power.

the distance and the orientation of the receiver coil to the transmitter are variable, resulting in different energy transfer rates. The wireless power transfer is zero when the cow stops eating and lifts her head, because the distance between transmitter and receiver becomes too large to continue energy transfer. This can be observed in Fig. 9a as horizontal plateaus. On the other hand, during some moments, the receiver is nearly optimally oriented, and the energy transfer is high. For example, between 22 and $34 \mathrm{~s}$ and between 43 and $50 \mathrm{~s}$, an average power transfer of 7.0 and $8.5 \mathrm{~W}$ is realized, respectively. A peak power transfer of $14 \mathrm{~W}$ (at $42 \mathrm{~s}$ ) was registered during this measurement. These higher momentary energy transfer rates validate the use of supercapacitors instead of a rechargeable Li-ion battery as energy buffer [24]. At 12 and $34 \mathrm{~s}$, the measurements indicate a small decrease in the energy stored due to a charge redistribution over the different supercapacitors because of different equivalent series resistances.

Fig. 9p shows a more typical measurement. During $124 \mathrm{~s}$, a total of $77 \mathrm{~J}$ was 


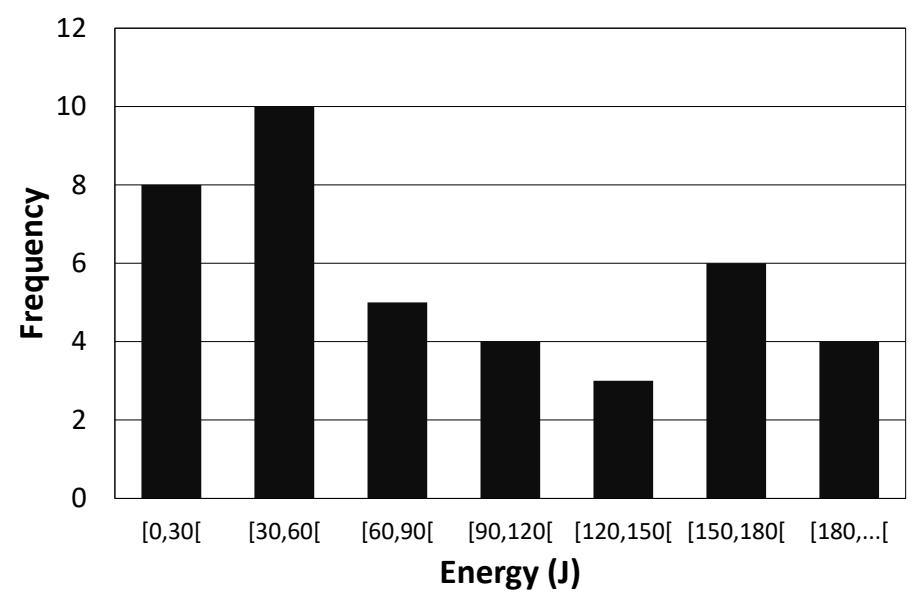

Figure 10: Distribution of the transferred energy per measurement.

transferred to the receiver, leading to an average of $0.62 \mathrm{~W}$. Some horizontal plateaus are present where the energy transfer is halted. For this measurement, the highest energy rate can be found between 58 and $74 \mathrm{~s}$, corresponding with $2.1 \mathrm{~W}$. This value is lower than the average power transfer of the measurement of Fig. 9a, indicating a less optimal orientation of the receiver than this previous measurement, even when the cow is eating.

To illustrate the great variety of the measurements,an example of a measurement with low power transfer is given: Fig. 9r shows a measurement where the cows eats almost continuously during $257 \mathrm{~s}$, with only short pauses. The energy transfer rate is fairly constant, at $0.11 \mathrm{~W}$. However, during the eating turn of more than 4 minutes, only $29 \mathrm{~J}$ is transferred, due to a tilted receiver coil during eating.

The average eating time over all 40 measurements was $160 \mathrm{~s}$. The shortest measurement lasted $49 \mathrm{~s}$, the longest $297 \mathrm{~s}$. Fig. 10 shows the distribution of the transferred energy per measurement. On average, an energy transfer of $96 \mathrm{~J}$ per meal was realized, with a minimum and maximum obtained value of 8 and $408 \mathrm{~J}$, respectively.

Fig. 11 shows that there is no correlation between the time the cow eats at the concentrate box and the amount of energy transferred to the receiver. 


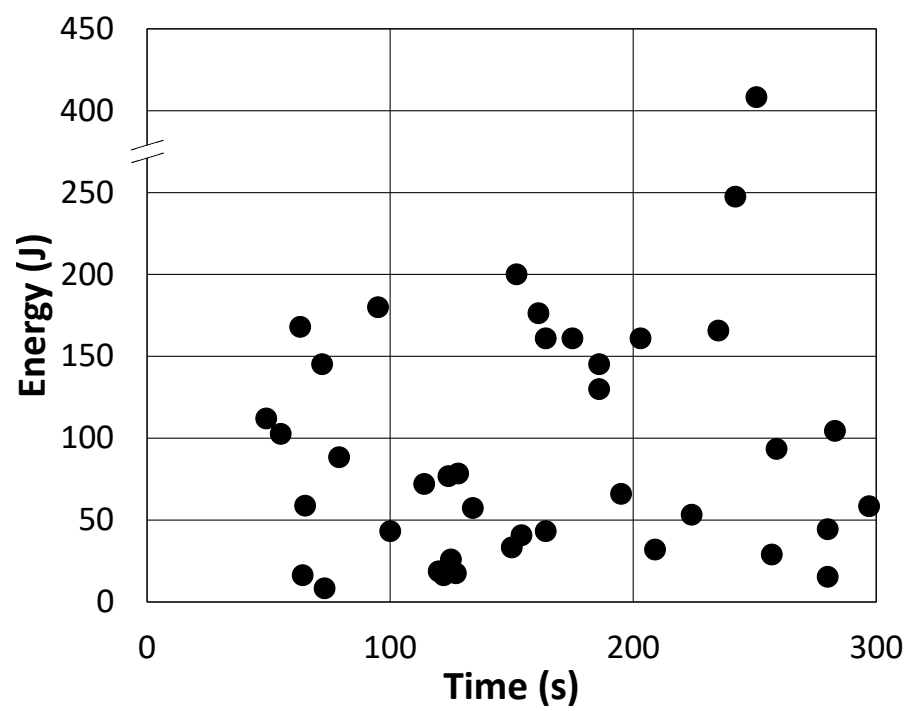

Figure 11: Overview of the energy transferred to the receiver as function of the time the cow stays at the concentrate feeding box for all the measurements.

Although this seems at first sight counter intuitive, this could be expected. When the receiver is badly oriented towards the transmitter, the energy transfer will be limited, whether or not the cow remains long at the feeding box. When the receiver is more optimally oriented, energy transfer is high, even if the eating time is limited. Moreover, a long time at the concentrate box does not indicate that the cow is continuously eating. The cow might be taken a lot of breaks from eating, while physically remaining at the concentrate box.

The variability of the measurements, due to the difference in behavior of each cow individually, is high. The standard deviations for the measurement times and transferred energy are $73 \mathrm{~s}$ and $80 \mathrm{~J}$, respectively. A sufficiently 320 large energy buffer can cover this variability, e.g., a hybrid configuration with supercapacitors as primary energy buffer, and Li-ion batteries as final energy storage for the system can allow continued operation of the system.

The cumulative frequency of the energy rate of the 40 different measurements is shown in Fig. 12. Even though a maximum average power transfer of $2.7 \mathrm{~W}$ was obtained for one measurement, the average power transfer of all 


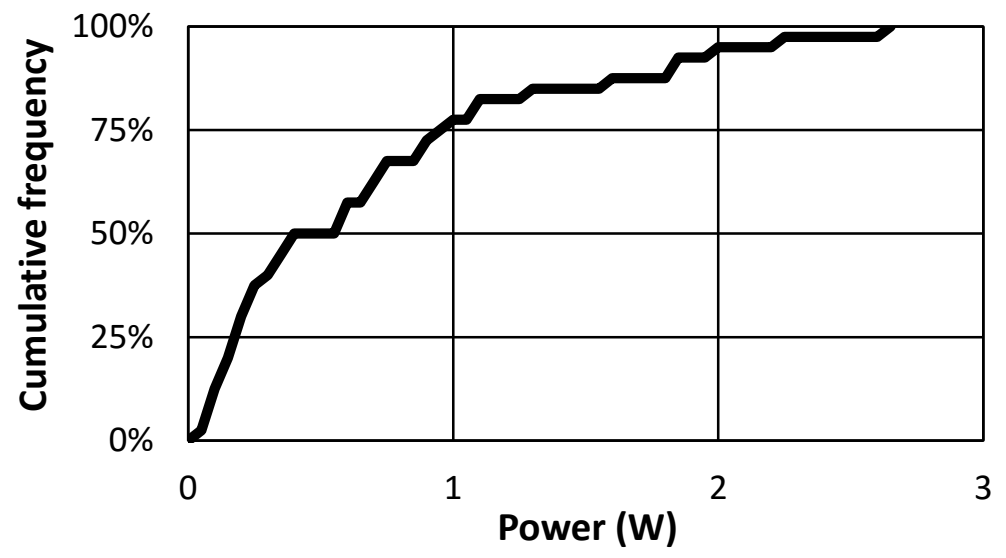

Figure 12: The cumulative frequency of the power transfer per measurement.

the measurements is about a factor four lower at $0.73 \mathrm{~W}$. The measurements with lower power transfer exceed the number of measurements with high power transfer (Fig. 12). The variability of the measurements is high with a standard deviation of $0.67 \mathrm{~W}$. The weighted average over time results in $0.60 \mathrm{~W}$.

\subsection{Practical implementation and future work}

Measurements at a research dairy farm resulted in an average energy transfer of $96 \mathrm{~J}$ per meal, for an average eating time of $160 \mathrm{~s}$. Existing cattle monitoring systems consume from about $5 \mathrm{~J}$ to $5 \mathrm{~kJ}$ of energy per day [8, 28, 29, 30], depending on their functionality, measured parameters, applied technology, and in particular the number of times a day data is transmitted and at which bit rate. Our results indicate that inductive wireless power transfer is a viable technology for certain applications to resolve the energy provision challenge for the automatic health monitoring of dairy cows.

The total time a dairy cow eats at a concentrate feeding box per day is highly variable and depends, among others, on the setup and organization of the farm, economical considerations and the dietary requirements of the animal [31, 32]. It is therefore difficult to make an estimation of the expected daily energy transfer for a wireless power transfer system installed at a concentrate feeding box. However, dairy cows often receive their concentrate in an automatic milking 
system would also be an ideal location for power transfer. The time a cow spends at an automatic milking system is less variable per farm than for a concentrate feeding box, allowing us to make a more reliable estimate for the daily energy transfer. Ketelaar-de Lauwere et al. 34 reports a total time from 35037 to 69 minutes per day per animal in an automatic milking system, distributed over 2.5 to 3 milking times a day [35. If the transmitter would be installed at an automatic milking system, an energy transfer of $1.3 \mathrm{~kJ}$ to $2.5 \mathrm{~kJ}$ per day could be expected, allowing the development of even more complex and integrated automatic health monitoring systems for dairy cows. However, it is possible that four different parameters: temperature, movement, position, and eating duration. This wireless power solution was developed in order to avoid battery replacements during the cow's lifetime. The collar of the cow contains the receiver coil for the wireless power transfer, but also serves as a central hub for tains an application processor for a first local analysis of the registered data, and two communication devices:

- An ultra-wideband radio for communication to a back end server.

- A near-field magnetic induction radio for communication with the lowpower temperature sensor, embedded in the ear-tag of the cow.

The experimental system has four primary goals:

- Detection of early signs of diseases, in particular fever and lameness. This can be realized by analyzing the activity of the cow (by the location sensor 
and, in second order, the accelerometer measurements), the temperature throughout the day, and the drinking frequency (determined by the cycles and duration of the presence of the cow at the drinking trough).

- Detection of heat, realized by mounting behavior (determined by the location sensor in the vertical axis and the accelerometer), social behavior (location sensor) and the cow calendar, coupled to a self-learning database on the back end server.

- Detection of birthing moment, based on sensor fusion of the temperature monitoring, cow calendar, and restless/social behavior.

- Location of the animal on the floor plan on request of the farmer (e.g., on his/her smartphone).

385

For this experimental system, an energy requirement of $0.2 \mathrm{~kJ}$ per $24 \mathrm{~h}$ for continuous operation of this system was determined, mainly attributed to the location tracking requirements. Given the results of this work, it seems feasible that the system can work without any battery replacements during the cow's lifetime. However, long-time field testing was not yet performed and the robustness and reliability of the system, including the wireless power transfer solution, is part of future research. Moreover, during certain time intervals, e.g., near the birthing moment, data should be collected and transmitted more frequently, which has its repercussions on energy consumption. More field tests are necessary to determine this impact.

\section{Conclusion}

A wireless power transfer system for an automatic health monitoring system for dairy cows was designed. By inductive coupling, the system was wirelessly charged every time the cow eats at a concentrate feeding box. The wireless link was optimized by choosing an elongated oval transmitter with ferrite present at transmitter and receiver side. Measurements at a research dairy farm resulted in an average energy transfer of $96 \mathrm{~J}$ per meal, for an average eating time of 
$160 \mathrm{~s}$. Our results indicate that inductive wireless power transfer is a viable technology to resolve the energy provision challenge for the automatic and realtime health monitoring of dairy cows. This can be considered as the removal of an important obstacle to increase profitability and efficiency for future farms. Long-time field testing to evaluate the robustness and reliability of the wireless power transfer system, in particular at automatic milking systems, is part of future research.

\section{Acknowledgment}

This work was executed within MoniCow, a research project bringing together academic researchers and industry partners. The MoniCow project was co-financed by imec (iMinds) and received project support from Flanders Innovation \& Entrepreneurship. We thank the Research Institute for Agriculture, Fisheries and Food Research for their assistance with the measurements at the dairy farm.

\section{References}

[1] B. Jones, "Growth in dairy farms: the consequences of taking big steps or small ones when expanding," in Four State Dairy Extension Conf., Wi and St. Paul, MN, 1999.

[2] L. W. Tauer and A. K. Mishra, "Dairy farm cost efficiency," J. of Dairy science, vol. 89, no. 12, pp. 4937-4943, 2006.

[3] L. Chase, L. Ely, and M. Hutjens, "Major advances in extension education programs in dairy production," J. of Dairy Science, vol. 89, no. 4, pp. 1147-1154, 2006.

${ }_{425}^{2}$ [4] "EU dairy farms - report 2013. based on FADN," European Union, 2014.

[5] T. Hemme, M. M. Uddin, and O. A. Ndambi, "Benchmarking cost of milk production in 46 countries," J. of Reviews on Global Economics, vol. 3, pp. 254-270, 2014. 
[6] J. Krieter, D. Cavero, and C. Henze, "Mastitis detection in dairy cows using neural networks." GIL Jahrestagung, vol. 101, pp. 123-126, 2007.

[7] J. Haugen, "Lameness in dairy cows in freestall barns with automatic milking systems," Ph.D. dissertation, 2011.

[8] H.F. Lopes, and N.B. Carvalho, "Livestock low power monitoring system," in Wireless Sensors and Sensor Networks (WiSNet), 2016 IEEE Topical Conference on, pp. 15-17.

[9] C. Arcidiacono, S.M.C. Porto, M. Mancino, and G. Cascone, "Development of a threshold-based classifier for real-time recognition of cow feeding and standing behavioural activities from accelerometer data," in Computers and Electronics in Agriculture, vol. 134, pp. 124-134, 2017.

[10] C.J. Rutten, C. Kamphuis, H. Hogeveen, K. Huijps, M. Nielen, and W. Steeneveld, "Sensor data on cow activity, rumination, and ear temperature improve prediction of the start of calving in dairy cows," in Computers and Electronics in Agriculture, vol. 132, pp. 108-118, 2017.

[11] M. Pastell, M. Kujala, A.M. Aisla, M. Hautala, V. Poikalainen, J. Praks, I. Veermäe, and J. Ahokas, "Detecting cow's lameness using force sensors," in Computers and Electronics in Agriculture, vol. 64, no. 1, pp. 34-38, 2008.

[12] S. Benaissa, F.A. Tuyttens, D. Plets, T. de Pessemier, J. Trogh, E. Tanghe, L. Martens, L. Vandaele, A. Van Nuffel, W. Joseph, and B. Sonck, "On the use of on-cow accelerometers for the classification of behaviours in dairy barns," in Research in Veterinary Science, 2017.

[13] S. Benaissa, D. Plets, E. Tanghe, G. Vermeeren, L. Martens, B. Sonck, F. A. M. Tuyttens, L. Vandaele, J. Hoebeke, N. Stevens et al., "Characterization of the on-body path loss at $2.45 \mathrm{GHz}$ and energy efficient wban design for dairy cows," IEEE Trans. Antennas Propag., vol. 64, no. 11, pp. 4848-4858, 2016. 
[14] S. Benaissa, D. Plets, E. Tanghe, L. Verloock, L. Martens, J. Hoebeke, B. Sonck, F. A. M. Tuyttens, L. Vandaele, N. Stevens et al., "Experimental characterisation of the off-body wireless channel at $2.4 \mathrm{GHz}$ for dairy cows in barns and pastures," Computers and Electronics in Agriculture, vol. 127, pp. 593-605, 2016.

[15] X. Lu, P. Wang, D. Niyato, D. I. Kim, and Z. Han, "Wireless charging technologies: Fundamentals, standards, and network applications," IEEE Commun. Surveys Tut., vol. 18, no. 2, pp. 1413-1452, 2016.

[16] X. Mou and H. Sun, "Wireless power transfer: Survey and roadmap," in Vehicular Technology Conf. (VTC Spring), 2015 IEEE 81st, 2015, pp. 1-5.

[17] S. Hui, "Wireless power transfer: A brief review \& update," in Power Electronics Systems and Applications (PESA), 2013 5th Int. Conf. on, 2013, pp. 1-4.

[18] T.-E. Stamati and P. Bauer, "On-road charging of electric vehicles," in Transportation Electrification Conf. and Expo (ITEC), 2013 IEEE, 2013, pp. 1-8.

[19] A. Pacini, F. Mastri, R. Trevisan, D. Masotti, and A. Costanzo, "Geometry optimization of sliding inductive links for position-independent wireless power transfer," in Microwave Symp. (IMS), 2016 IEEE MTT-S Int., 2016, pp. 1-4.

[20] M. Simic, C. Bil, and V. Vojisavljevic, "Investigation in wireless power transmission for uav charging," Procedia Computer Science, vol. 60, pp. 1846-1855, 2015.

[21] M. J. Chabalko, M. Shahmohammadi, and A. P. Sample, "Quasistatic cavity resonance for ubiquitous wireless power transfer," PloS one, vol. 12, no. 2, p. e0169045, 2017.

[22] B. Thoen, S. Wielandt, J. De Baere, J.-P. Goemaere, L. De Strycker, and N. Stevens, "Design of an inductively coupled wireless power system for 
moving receivers," in Wireless Power Transfer Conf. (WPTC), 2014 IEEE, 2014, pp. 48-51.

[23] K. Van Schuylenbergh and R. Puers, Inductive powering: basic theory and application to biomedical systems. Springer Science \& Business Media, 2009.

[24] B. Minnaert, B. Thoen, D. Plets, W. Joseph, and N. Stevens, "Optimal energy storage solution for an inductively powered system for dairy cows," in Wireless Power Transfer Conf. (WPTC), 2017 IEEE. 2017, pp. 1-4.

[25] J. Kim, J. Kim, S. Kong, H. Kim, I.-S. Suh, N. P. Suh, D.-H. Cho, J. Kim, and S. Ahn, "Coil design and shielding methods for a magnetic resonant wireless power transfer system," Proc. IEEE, vol. 101, no. 6, pp. 1332-1342, 2013.

[26] S. Wielandt and N. Stevens, "Influence of magnetic design choices on the quality factor of off-the-shelf wireless power transmitter and receiver coils," in Wireless Power Transfer Conf. (WPTC), 2013 IEEE. 2017, pp. 151-154.

[27] K.M. Havstad and K.M. Olson-Rutz, "Sample size determinations for studying selected cattle foraging behaviors," in Applied Animal Behaviour Science, Vol. 30, pp. 17-26, 1991.

[28] I. Andonovic, C. Michie, M. Gilroy, H.G. Goh, K.H. Kwong, K. Sasloglou and T. Wu, "Wireless sensor networks for cattle health monitoring," in ICT innovations 2009, pp. 21-30, 2010.

[29] A. Kumar, G.P. and Hancke, "A zigbee-based animal health monitoring system," in IEEE sensors Journal, vol. 15, no. 1, pp. 610-617, 2015.

[30] K.H. Kwong, T.T. Wu, H.G. Goh, K. Sasloglou, B. Stephen, I. Glover, C. Shen, W. Du, C. Michie, and I. Andonovic, "Practical considerations for wireless sensor networks in cattle monitoring applications," in Computers and Electronics in Agriculture, vol. 81, pp. 33-44, 2012. 
[31] J. Van Geneijgen and A. Smits, Waiboerhoeve 1976. Proefstation voor de rundveehouderij, 5 1977, ch. 7, pp. 41-46.

[32] R. Kellaway and T. Harrington, Feeding concentrates: supplements for dairy cows. Landlinks Press, 2004.

[33] C. Ketelaar-de Lauwere, S. Devir, and J. Metz, "The influence of social hierarchy on the time budget of cows and their visits to an automatic milking system," Applied Animal Behaviour Science, vol. 49, no. 2, pp. 199-211, 1996.

[34] C. Ketelaar-de Lauwere, M. Hendriks, J. Zondag, A. Ipema, J. Metz, and J. Noordhuizen, "Influence of routing treatments on cows' visits to an automatic milking system, their time budget and other behaviour," Acta Agriculturae Scandinavica, Section A-Animal Science, vol. 50, no. 3, pp. 174-183, 2000.

[35] K. de Koning and J. Rodenburg, "Automatic milking: State of the art in 525 europe and north america," in Automatic milking: A better understanding. Wageningen Academic Publishers, Wageningen, the Netherlands, 2004, pp. 27-37. 